\title{
A new shock factor of SWATH catamaran subjected to underwater explosion
}

\section{Abstract}

In order to measure the extreme degree of the impact environment on a SWATH (small waterplane area twin hull) catamaran subjected to an underwater explosion, this work defines a new type of shock factor based on shock wave energy. This new shock factor is used as a parameter to describe the response from this kind of ship, and it is divided into two categories according to characteristics of the SWATH catamaran structure. This research used finite element software to simulate and calculate the response of SWATH catamaran subjected to an underwater explosion. The validity of this new shock factor is proved in three categories, including total kinetic energy, total strain energy and shock spectrum. The results show that the responses of the SWATH catamaran structure are similar when the new shock factor is equal, and it is also appropriate for situations involving short explosive distances.

Keywords: SWATH catamaran; Underwater explosion; New shock factor; Impact environment

\section{Introduction}

With the development of various anti-ship weapons, a ship's ability to resist load impact has gained more attention. In previous naval warfare and underwater explosion shock tests, the results have shown that the ships share a common weak link in that they lack the ability to resist load impacts. As such, there is significant research 
required to determine the impact environment of a ship's structure. When compared with a monohull ship, the SWATH catamaran, as a newly developing warship, has a number of advantages, including fast route speed, good stability and low resistance, and it has been gradually implemented into naval use [1]. In 1969, the Dutch built the first semi-submersible ship SWATH catamaran "DUPLUS" with a full displacement of 1,200t and a speed of 9 knots [2]. After that, the United States built the "KAIMALINO" research vessel [3, 4]; that ship was launched in 1973 and is still in service. The United States has also built several "VICTORY" class SWATH catamarans as monitors. At the same time another naval power, Germany, introduced the SWATH construction technology from the United States and produced 14 SWATH vessels, which are used for water monitoring, hydrological monitoring and anti-submarine and other military expedition auxiliary tasks. With the popularity of using SWATHs for military purposes, its anti-explosion and anti-shock performance has received increasing attention from various navies. The shock factor is a parameter that is widely used for the macroscopic representation of a ship's impact environment. There are three kinds of shock factors: 1) shock factor $C_{1}$, which is based on the hypothesis of a higher-pressure shock wave; it can be obtained from the experience formula of the Cole[5]. If there are two different conditions of the equal impact factor $C_{1}$, it is considered that the higher pressure of shock waves are equal at the incident point; this form factor has been used for the submarines structure's vitality assessment by NATO and the former Soviet Union[6]. In earlier forms of the impact factor computations, a large number of calculations show that the peak pressure generated 
by a small explosive in the near field can sometimes be larger than the large explosive in the far field, but any damage done to the ship's structure is not significant; 2) the shock factor $C_{2}$ is based on the plane wave hypothesis [7]; this definition comes from the viewpoint of energy that has been acted upon the structure. Studies have shown that $C_{2}$ can reflect the similarity of an underwater explosion load in the far field, but the plane wave hypothesis will not be useful in the near field; 3) when applied to monohull ships the new shock factor $C_{3}[8]$ has relatively high accuracy, but is only appropriate for monohull ships. Consequently, a new shock factor must be defined to describe the similarity of the impact environment on the SWATH catamaran. We have designed a new shock factor based on the concept of the monohull ship's shock factor $\mathrm{C}_{\text {cata. }}$, which we applied to a SWATH catamaran.

\section{Theoretical analysis}

2.1 Interaction between the shock wave and the hull

When the ship is subjected to an underwater explosion, it is affected by the shock wave. The shock wave propagates in various directions in the form of a spherical wave; the shock wave energy is then distributed in the whole spherical wavefront while the ship is in an infinite field. In the point of energy, the underwater explosion radiates energy to the area surrounding it, which is similar to a light source. This work presents three hypotheses for the convenience of research in this paper [7]:

(1) The diffraction pressure of the shock wave acting on the structure is regarded as a secondary factor, and is not considered in our calculations; 
(2) The energy dissipation of the shock wave is at its most serious around the explosive. When the distance is beyond twenty-five times the explosive's radius, the energy dissipation is thought to be very slow. The explosion distance is well over twenty-five times the radius in this paper, so the energy dissipation is not given specific consideration.

(3) The differences in shock wave reflectivity between the near-field and far-field structure have not been included.

Assuming that the explosive is W kilograms TNT while the chemical energy per unit mass is $\rho_{e}$, and the conversion rate of the chemical energy and the shock wave energy is $\eta_{e}$, the explosion energy $E$ released by $\mathrm{W}$ kilograms explosive is as follows:

$$
E=W \rho_{\mathrm{e}} \eta_{\mathrm{e}}
$$

If the explosion distance is long, we assume that the shock wave impacts the hull structure in the form of a plane wave; the shock wave energy can be calculated using the following formula:

$$
E_{\mathrm{e}}=\frac{W \rho_{\mathrm{e}} \eta_{\mathrm{e}} S_{\mathrm{e}}}{4 \pi R^{2}}=\frac{\rho_{\mathrm{e}} \eta_{\mathrm{e}} S_{\mathrm{e}}}{4 \pi} \cdot \frac{W}{R^{2}}=K C_{2}^{2}
$$

If the position of the explosion is fixed, the value of shadow area $S_{e}$ is unchanged and a constant; the variable is only $W / R^{2}$, which is the shock factor $C_{2}$ based on the traditional plane wave hypothesis. The reason for this is that the shock wave spreads in the form of a spherical wave, but the plane wave hypothesis is 
only applicable to long explosion distances. To get a more accurate calculation of shock wave energy, we need to consider the characteristics of the spherical shock wave:

$$
E_{\mathrm{e}}=\frac{W \rho_{\mathrm{e}} \eta_{\mathrm{e}}}{4 \pi R^{2}} \cdot S_{\mathrm{e}}=\frac{\rho_{\mathrm{e}} \eta_{\mathrm{e}}}{4 \pi} \cdot \frac{W}{R^{2}} \cdot S_{\mathrm{e}}
$$

Even though the position of the explosion is fixed, if $W$ or $R$ is unchanged, $S_{e}$ is also changed. The shock wave energy that acts on the hull includes the variable $W$, $R$ and $S_{e}$. As such, it is important to accurately obtain the shock wave energy impacting the hull. Not only are $W$ and $R$ certain, but the value of $S_{e}$ must also be calculated precisely.

2.2 Calculation of shadow area based on spherical wave theory

Assuming there is a random smooth and continuous curved surface in space, (shown in Fig. 2.1) the energy from the coordinate's origin (explosion position) will not pass through this curved surface twice [9]. Defining the infinitesimal area in the curved surface is $d S$, and the distance of the infinitesimal area and the origin is defined as the explosion distance $R$. The projected area of $d S$ on spherical surface with the radius $R$ is thus:

$$
d S_{R}=d S \cdot \cos \gamma
$$




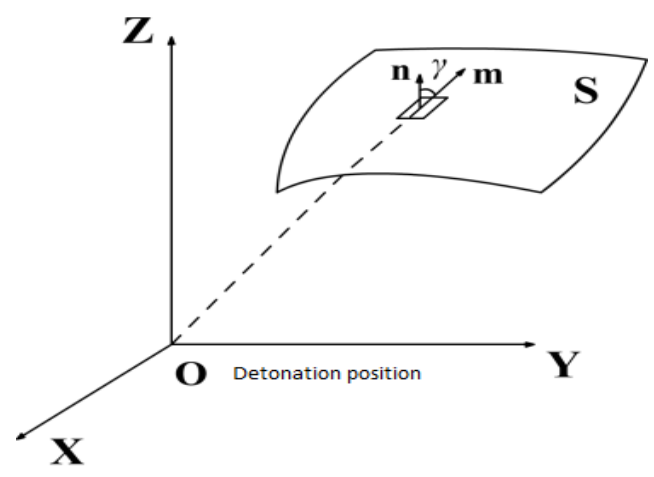

Fig. 2.1 Shadow area of the space curved surface

Where $\gamma$ is the included angle between the normal vector $\vec{n}$ and the radius vector $\vec{m}$. Then the infinitesimal area $d S$ is obtained in spherical coordinate as:

$$
d S_{R}=R d \varphi \cdot R \sin \varphi d \theta
$$

The shadow ratio of the shock wave sheltered by the infinitesimal area is defined as:

$$
\eta=\frac{\iint_{s} d S_{R}}{4 \pi R^{2}}=\frac{\iint_{s} R^{2} \sin \varphi d \varphi d \theta}{4 \pi R^{2}}=\frac{1}{4 \pi} \iint_{s} \sin \varphi d \varphi d \theta
$$

The expression shows that the calculation of the shadow ratio $\eta$ is independent of the selection of the projected spherical surface, which is determined by the limits of the integration of $\varphi$ and $\theta$. The shadow area is given by:

$$
S_{e}=4 \pi R_{0}^{2} \eta
$$

2.3 The finite element method

In this paper, the finite element method is used to verify the correctness of the $d S$. Models are built in ANSYS, and the wet surface of structures is made mesh using 
ABAQUS and the output INP file. These steps are listed in Fig. 3.1[9]:

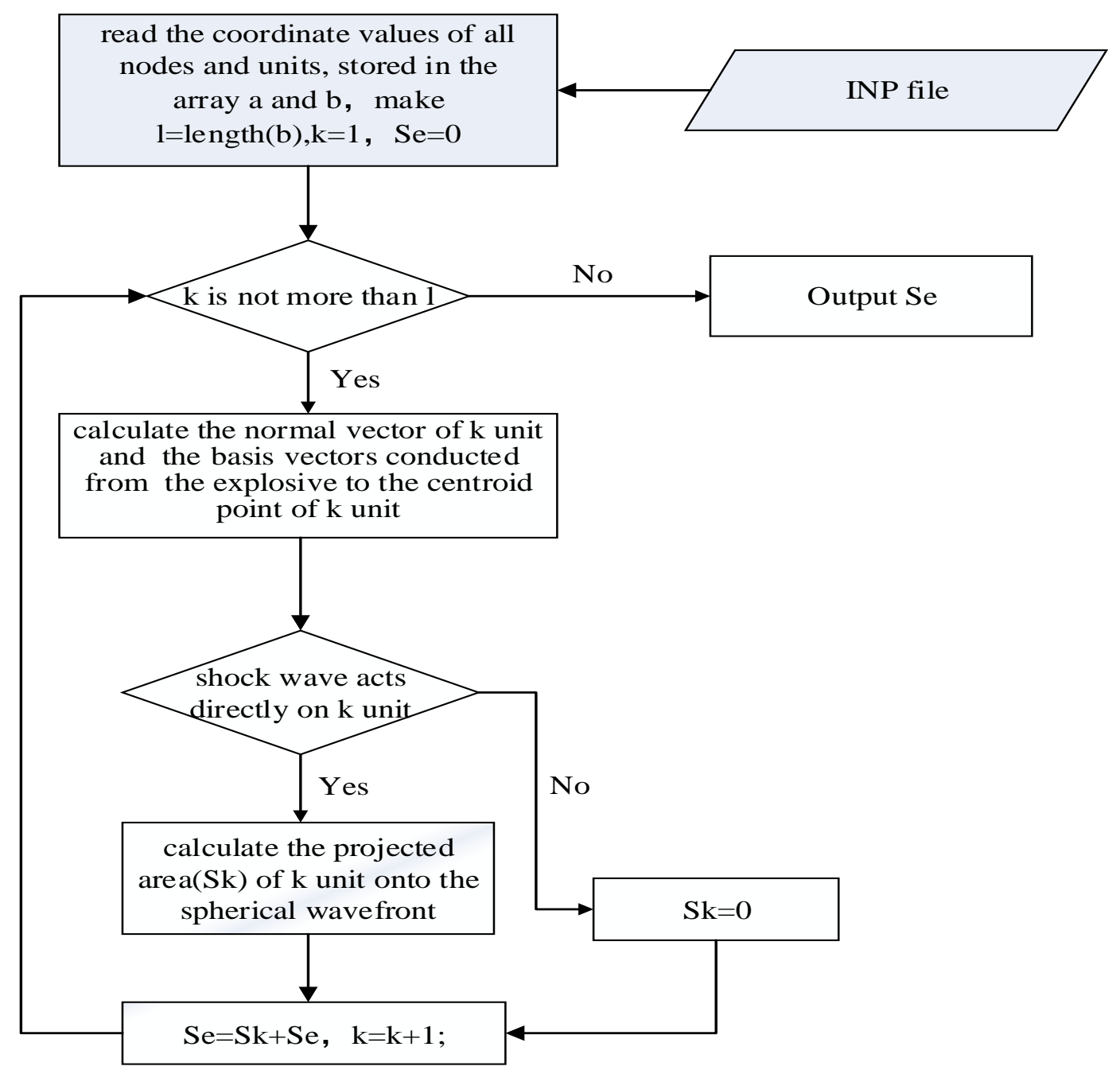

Fig. 3.1 The MATLAB program flowchart

Therefore, this MATLAB program can be used to verify the accuracy of the calculation formula for the shadow area.

\section{The new shock factor}

According to the expression (3), the new shock factor applied to the SWATH catamaran is defined as $\mathrm{C}_{\text {cata. }}=\sqrt{\mathrm{S}_{\mathrm{e}} W} / R$, and the approximate calculation formula for the shadow area $S_{e}$ can be obtained according to expressions (6) and (7), using the finite element method afterward to verify the accuracy and then to modify, thus 
obtaining the new shock factor applied to SWATH catamaran.

\subsection{The theoretical calculation}

Because the catamaran's underwater structure is complex and not easy to analyze, this paper sets equal and simplified models to replace the underwater structure. Models A and B have been applied to replace the underwater structure based on the characteristic of a catamaran's underwater structures. Model A is similar to the underwater structure of a catamaran and the draft is equal to the real model. The cylinder diameter of Model B is equal to the draft of the real model. The simplified models are in the following figures:

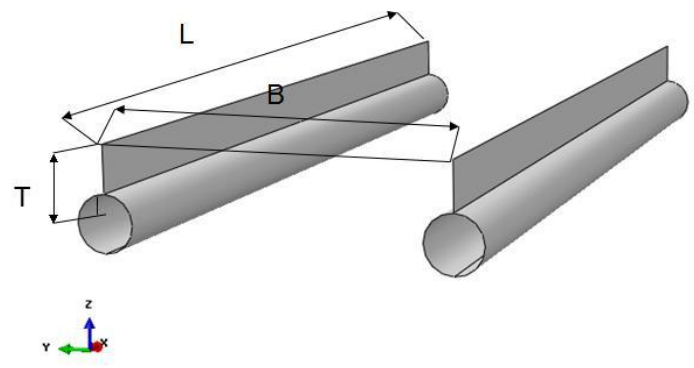

(a) Model A

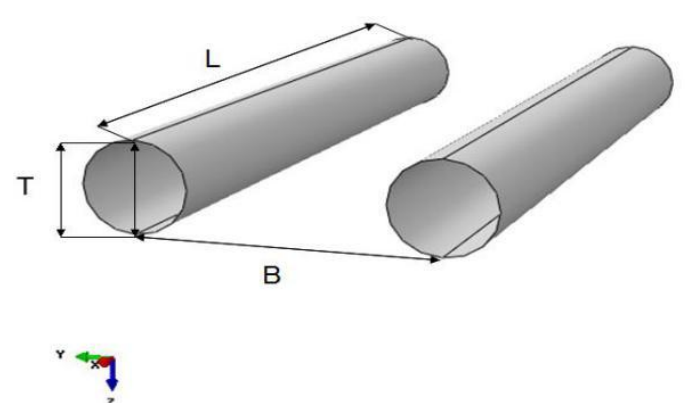

(b) Model B

Fig. 3.2 The simplified model

First, the finite mesh of the models were divided; then different conditions were chosen in which $W, R, \theta$ are equal; the $S_{e}$ of the models were then calculated by using spherical wave made by MATLAB.

The principal dimensions of the SWATH are as follows: the length is $60 \mathrm{~m}$; the 
width is $20 \mathrm{~m}$; the depth is $6 \mathrm{~m}$, the full load is about 1300t. Models were built in ANSYS, and the hull coordinate system with the Z-axis is for the draft direction, and $\mathrm{Z}$ moves upward as it increases; the $\mathrm{X}$-axis is for the width direction, the port is the direction of increasing $\mathrm{X}$; the $\mathrm{Y}$-axis is for the length direction, the bow is the direction of increasing Y. The finite element model is shown in Fig. 3.3:

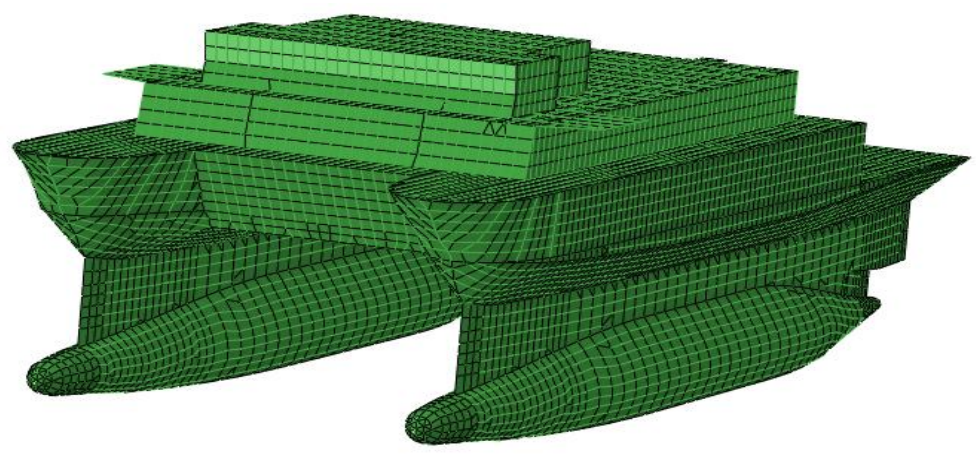

Fig. 3.3 The finite element model of the SWATH

The flow field in the finite element model was established using an acoustic unit. The geometry of the flow field is a combination of a half cylinder and a hemisphere. The hemisphere's diameter is about 6 times the width of the ship [10], and the hull is embedded and coupled in the flow field; the acoustic-structure coupling method was used to simulate an underwater explosion. The schematic diagram of the finite element model for the coupling of the hull and the flow field is shown in Fig. 3.4. 


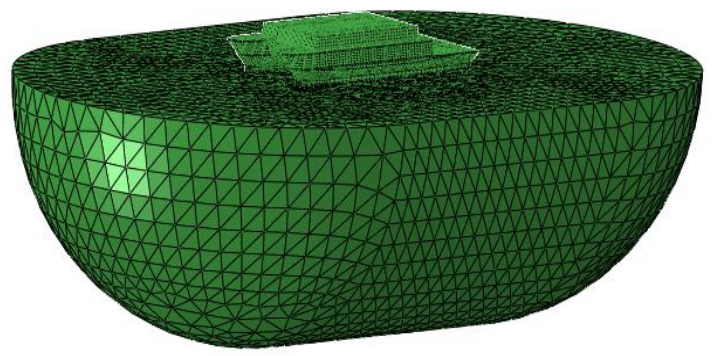

Fig. 3.4 The schematic diagram for the coupling of the hull and the flow field

The mesh size has a great influence on the accuracy of the calculation. However, the flow unit grid plays a leading role in underwater explosions [10].The maximum length of the unit is determined using the upper limit frequency of the shock wave, as follows:

$$
L_{\max }<\frac{c}{n^{*} f_{\max }}
$$

where $c$ is the wave speed of the material, $n$ is the number of the unit in the length of each shock wave, generally not less than $6 ; f_{\max }$ is the maximum frequency of the shock wave, defined as the inverse of the pulse width. In this paper, $f_{\max }$ is $250 \mathrm{~Hz}$; the minimum of $c$ is $4500 \mathrm{~m} / \mathrm{s} ; n$ is no less than 20 . Based on the above requirements, the size of the fluid and the structural unit is $0.1 \mathrm{~m}$.

Below are some calculations in different conditions. The shadow area is shown in the following figure in which the spherical cartridge is $40 \mathrm{~m}$ directly below the mid ship. 


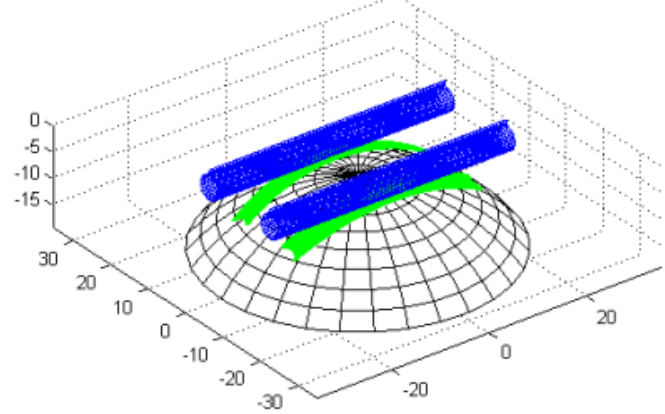

(a) The shadow area of model A

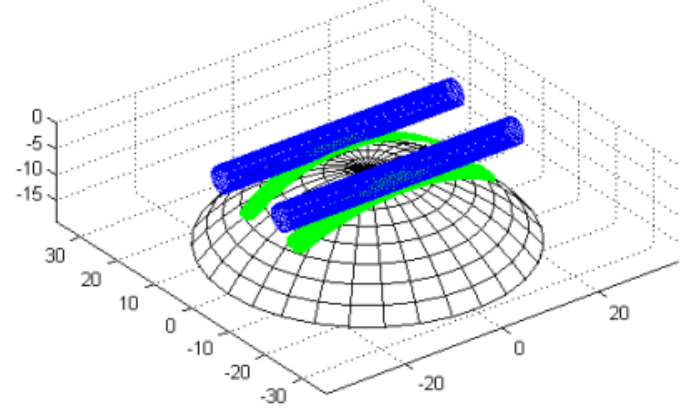

(b) The shadow area of model B

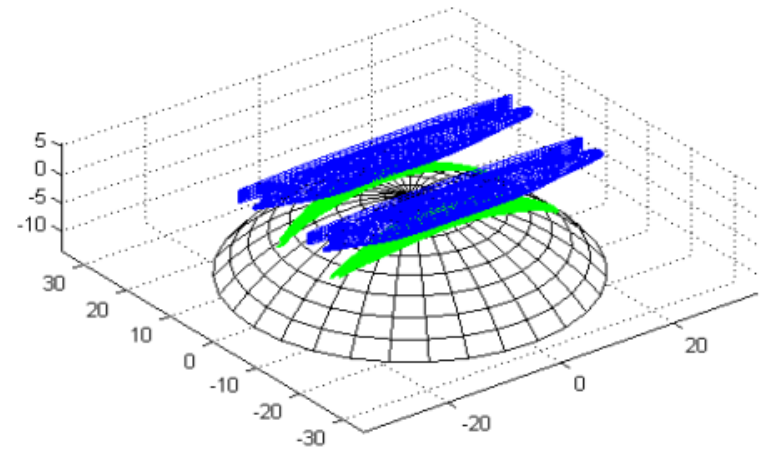

(c)The real shadow area of the SWATH

Fig. 3.5 The shadow area of a different model

Table 3.1 the shadow area of different models $\left(\mathrm{m}^{2}\right)$

\begin{tabular}{l|c|c|c|c}
\hline Model & $\mathrm{A}\left(\mathrm{m}^{2}\right)$ & $\mathrm{B}\left(\mathrm{m}^{2}\right)$ & $\mathrm{SWATH}\left(\mathrm{m}^{2}\right)$ & the error $(\%)$ \\
\hline $1-1$ & 290.4 & 408.055 & 397.9 & 2.6 \\
\hline $1-2$ & 269.5 & 367.556 & 388.5 & 5.3 \\
\hline
\end{tabular}


Note: Condition 1-1 is one in which the spherical cartridge is $40 \mathrm{~m}$ directly below the midship; condition $1-2$ is $60 \mathrm{~m}$ to the right of midship section with the water at a depth of $40 \mathrm{~m}$. The error is model B compared to the SWATH.

The result shows that: Model B was established based on the principle that equal draft can replace the underwater structure of the SWATH catamaran [11]. The structure shown in Fig. 3.6 is the real underwater structure of the SWATH catamaran, and Fig. 3.7 shows a simplified finite element model.
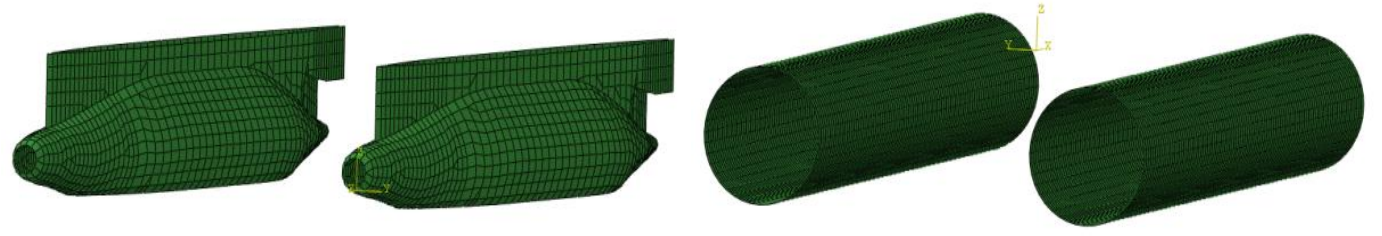

Fig. 3.6 The real underwater structure Fig. 3.7 The simplified finite element model

Because the underwater structure of the SWATH catamaran is non-continuous, the shadow area of shock wave energy varies as the explosive's lateral position changes. At first, it needs to judge the condition of the shadow before calculating $S_{e}$. When the longitudinal coordinate of the explosive is constant, the shadow area of the shock wave energy varies according to the lateral and vertical coordinates of the explosive. Using the boundary element method [12], the condition of the shadow is divided into two categories for the purposes of this paper (shown in Fig. 3.8).

The first shadow condition: The explosive changes between the point $O$ and the critical point $O^{\prime}$

The second shadow condition: The explosive is to the right of the critical point. 


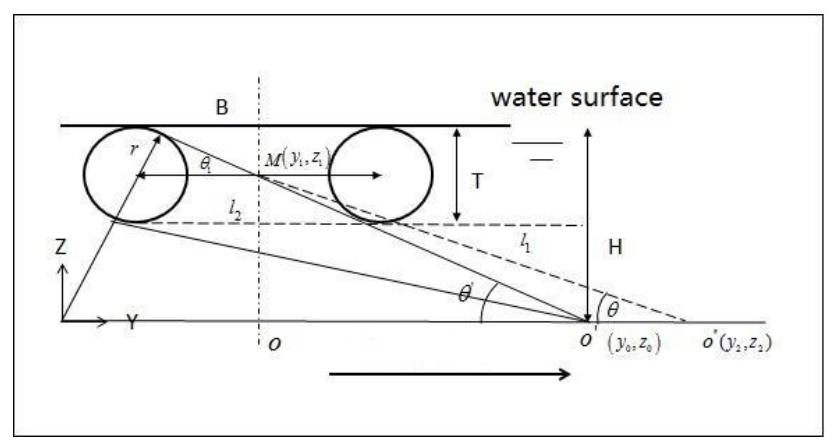

Fig. 3.8Schematic diagram of the shadow condition

When the energy particle radial is tangent to the left and right cylinders, it is defined as the critical condition, and the angle $\theta^{\prime}$ is the critical angle. We assume that the midpoint $M$ of the connection line between these two cylindrical centers is an auxiliary point, and its coordinate are (y1, z1). The angle $\theta$ has an invariable expression according to the coordinate relationship:

$$
\theta=\arctan \left|\frac{z_{2}-z_{1}}{y_{2}-y_{1}}\right|
$$

When $\theta<\theta^{\prime}$, it means the explosion center isout of the critical point. The angle $\theta$ can be obtained by the geometric relationship:

$$
\theta=\arctan \left|\frac{z_{2}-z_{1}}{y_{2}-y_{1}}\right|<\theta^{\prime}=\arcsin 2 r / B
$$

When $\theta$ is coincident with the expression (9), this indicates the second shadow condition, otherwise it indicates the first shadow condition. According to the shadow condition and using the expression (6) to calculate the shadow area of the cylinder, in order to obtain an expression that is suitable to the shock factor, we must ignore the changes at the end of the cylinder of the integration limits: 


$$
\eta_{c y l}=\frac{1}{4 \pi}\left(\int_{\beta_{1}}^{\beta_{2}} \int_{\alpha_{1}}^{\alpha_{2}} \sin \varphi d \varphi d \theta+\int_{\beta_{3}}^{\beta_{4}} \int_{\alpha_{3}}^{\alpha_{4}} \sin \varphi d \varphi d \theta\right)
$$

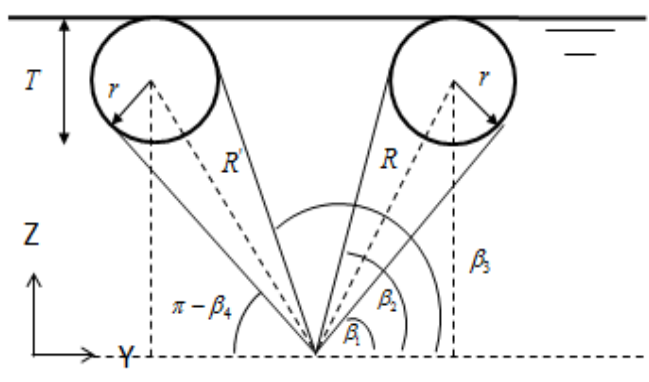

Fig. 3.9 Cross-section chart of the first shadow condition

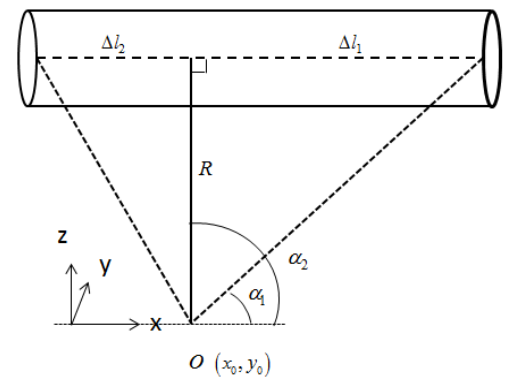

Fig. 3.10 Side-sectional chart of the first shadow condition

Fig. 3.9 and Fig. 3.10 are the section charts of the first shadow condition, $\beta_{\mathrm{i}} 、 \alpha_{\mathrm{i}}(i=1,2,3,4)$ are different with $\eta$ changes, and the expression is as follows according to the geometric relationship:

$$
\begin{array}{cc}
\beta_{1}=\arcsin \frac{H-r}{r+R}-\arcsin \frac{r}{r+R} & \beta_{2}=\arcsin \frac{H-r}{r+R}+\arcsin \frac{r}{r+R} \\
\beta_{3}=\pi-\left(\arcsin \frac{H-r}{r+R^{\prime}}+\arcsin \frac{r}{r+R^{\prime}}\right) & \beta_{4}=\pi-\arcsin \frac{H-r}{r+R^{\prime}}+\arcsin \frac{r}{r+R^{\prime}} \\
\alpha_{1}=\frac{\pi}{2}-\arctan \frac{\Delta l_{1}}{R} & \alpha_{2}=\frac{\pi}{2}+\arctan \frac{\Delta l_{2}}{R} \\
\alpha_{3}=\frac{\pi}{2}-\arctan \frac{\Delta l_{1}}{R^{\prime}} & \alpha_{4}=\frac{\pi}{2}+\arctan \frac{\Delta l_{2}}{R^{\prime}}
\end{array}
$$

Where $H$ represents the vertical distance from the water surface and the explosion position, $r$ is the radius of the cylinder of the simplified model; $R$ is the distance of the explosion position and the right side of the cylinder. $R$ ' is the distance of the explosion position and the left side of the cylinder; $\Delta l_{1}$ and $\Delta l_{2}$ are the longitudinal distances of the explosion position and the end of the model. The shadow 
area of the first condition can be obtained using the above expression:

$$
S_{e 1}=2 R^{2}\left(\arcsin \left(\frac{r}{r+R}\right)\left(\sin \left(\arctan \frac{\Delta l_{2}}{R}\right)+\sin \left(\arctan \frac{\Delta l_{1}}{R}\right)\right)+\arcsin \left(\frac{r}{r+R}\right)\left(\sin \left(\arctan \frac{\Delta l_{2}}{R}\right)+\sin \left(\arctan \frac{\Delta l_{1}}{R^{\prime}}\right)\right)\right)
$$

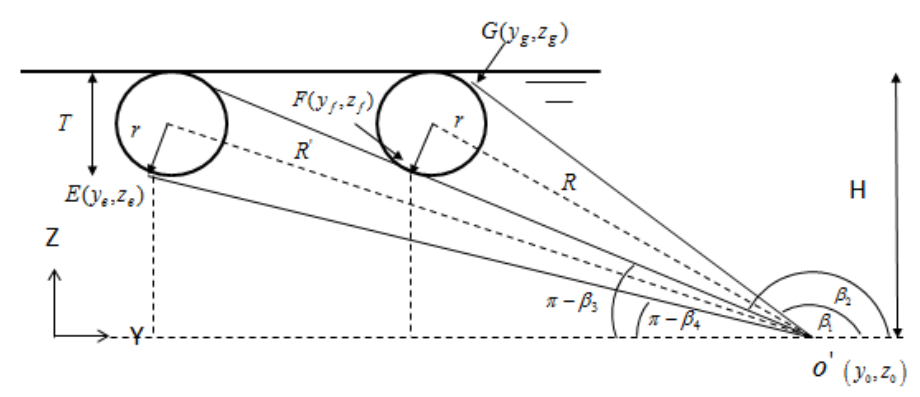

Fig. 3.11 Cross-section schematic of the second shadow condition

Expression (9) can be used as the shock factor of the second shadow condition (shown in Fig. 3.11), with the exception that the calculation formula for the upper and lower integral limits are different from the first condition, and there is:

$$
\begin{aligned}
& \beta_{1}=\pi-\left(\arctan \left|\frac{z_{f}-z_{o}}{y_{f}-y_{o}}\right|+2 \arcsin \frac{r}{r+R}\right) \\
& \beta_{2}=\beta_{3}=\pi-\arctan \left|\frac{z_{f}-z_{o}}{y_{f}-y_{o}}\right| \\
& \beta_{4}=\pi-\arctan \left|\frac{z_{e}-z_{o}}{y_{e}-y_{o}}\right| \\
& \alpha_{1}=\frac{\pi}{2}-\arctan \frac{\Delta l_{1}}{R} \alpha_{2}=\frac{\pi}{2}+\arctan \frac{\Delta l_{2}}{R}
\end{aligned}
$$




$$
\alpha_{3}=\frac{\pi}{2}-\arctan \frac{\Delta l_{1}}{R^{\prime}} \alpha_{4}=\frac{\pi}{2}+\arctan \frac{\Delta l_{2}}{R^{\prime}}
$$

Then the shadow area of the second condition can be obtained:

$$
S_{e 1}=R^{2}\left(\begin{array}{l}
\arcsin \left(\frac{r}{r+R}\right)\left(\sin \left(\arctan \frac{\Delta l_{2}}{R}\right)+\sin \left(\arctan \frac{\Delta l_{1}}{R}\right)\right)+ \\
\left(\arctan \left|\frac{z_{e}-z_{0}}{y_{e}-y_{0}}\right|-\arctan \left|\frac{z_{f}-z_{0}}{y_{f}-y_{0}}\right|\right)\left(\sin \left(\arctan \frac{\Delta l_{2}}{R^{\prime}}\right)+\sin \left(\arctan \frac{\Delta l_{1}}{R^{\prime}}\right)\right)
\end{array}\right)
$$

\subsection{Amending the shadow area with the finite element method}

Because the calculation formula for the shadow area of the cylinder is approximate, it needs to verify the accuracy of the theoretical formulas for two shadow conditions. In this part, the theoretical approximate result is compared with the result obtained from the finite element method, and is used to amend the condition with a large error [13]. To ensure that there is a single variable during the verification process it is necessary to change the value of $r / L$ by changing the value of the draft $(\mathrm{T}=2 \mathrm{r})$ and the length respectively, and then change the explosion position[14]. The explosive is $20 \mathrm{~m}, 30 \mathrm{~m}, 40 \mathrm{~m}, 50 \mathrm{~m}$ directly below the midship; the quality of the explosive is $9.6 \mathrm{Kg}, 29.7 \mathrm{Kg}, 35 \mathrm{Kg}, 44.3 \mathrm{Kg}$. The contrast results of the first shadow condition are shown in Table 3.1 and Table 3.2:

\begin{tabular}{|c|c|c|c|c|c|c|c|c|c|c|}
\hline Explosion & & & & & & $/ \mathrm{L}$ & & & & \\
\hline $\begin{array}{l}\text { Distance } \\
\text { (m) }\end{array}$ & 5.45 & 7.27 & 9.09 & 10.91 & 12.73 & 14.55 & 16.36 & 18.18 & 20.00 & 21.82 \\
\hline
\end{tabular}

Table $3.2 \mathrm{~T}=5.5 \mathrm{~m}$, the error value corresponding to different $\mathrm{r} / \mathrm{L}, \mathrm{R}(\%)$ 


\begin{tabular}{c|c|c|c|c|c|c|c|c|c|c}
\hline 16.7 & 1.8 & 1.5 & 1.1 & 1.4 & 1.0 & 0.7 & 0.4 & 1.1 & 1.1 & 1.0 \\
\hline 25.9 & 1.6 & 1.4 & 1.3 & 1.2 & 1.1 & 1.1 & 1.0 & 1.2 & 1.2 & 1.1 \\
\hline 35.6 & 1.4 & 1.2 & 1.1 & 1.4 & 1.1 & 1.3 & 1.2 & 1.4 & 1.4 & 1.3 \\
\hline 45.3 & 1.1 & 1.1 & 1.1 & 1.3 & 1.2 & 1.3 & 1.2 & 1.6 & 1.5 & 1.5 \\
\hline
\end{tabular}

Table 3.3 $\mathrm{L}=60 \mathrm{~m}$, the error value corresponding to different $\mathrm{r} / \mathrm{L}, \mathrm{R}(\%)$

\begin{tabular}{c|c|c|c|c|c|c|c|c|c|c}
\hline \multirow{2}{*}{ Vertical } & \multicolumn{7}{c}{ r/L } \\
\cline { 2 - 11 }$(\mathrm{m})$ & 10.00 & 10.91 & 12.00 & 13.33 & 14.12 & 15.00 & 16.00 & 17.14 & 18.46 & 20.00 \\
\hline-20 & 1.0 & 1.1 & 1.1 & 1.0 & 1.0 & 0.8 & 0.8 & 0.8 & 1.1 & 1.0 \\
\hline-30 & 1.0 & 1.0 & 0.9 & 1.2 & 1.1 & 0.9 & 0.9 & 0.8 & 1.1 & 1.1 \\
\hline-40 & 1.2 & 1.2 & 1.1 & 1.0 & 0.9 & 1.2 & 1.1 & 1.1 & 1.9 & 1.9 \\
\hline-50 & 1.2 & 1.1 & 1.0 & 1.1 & 1.1 & 1.4 & 1.4 & 1.4 & 1.9 & 1.9 \\
\hline
\end{tabular}
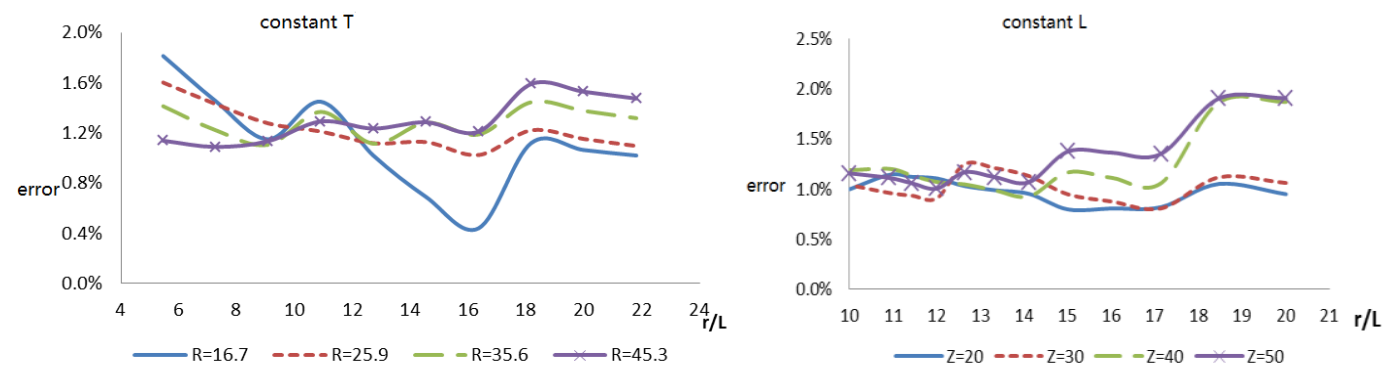
(a) Draught is unchanged (b) Longitudinal length is unchanged

Fig. 3.12 Graph of the error changing with $\mathrm{r} / \mathrm{L}$

The graphs based on the data in Tables 3.2 and 3.3 are shown in Fig. 3.11. As shown in Fig. 3.12 (a), the error is almost unchanged with the $r / L$ changing when the explosion distance is a constant; however, when the explosion distance is $16.7 \mathrm{~m}$, there is large fluctuation and the range is $0.4 \% \sim 1.8 \%$. As shown in Fig. 3.12 (b), when 10 $<\mathrm{r} / \mathrm{L}<15$, the error is almost unchanged with the $\mathrm{r} / \mathrm{L}$ changing, and it is not affected by the explosion distance. When $14<\mathrm{r} / \mathrm{L}<20$, the error increases as the explosion distance increases. When $\mathrm{z}=-50, \mathrm{r} / \mathrm{L}=20$, the maximum error is $1.9 \%$. In summary, the corresponding error values are less than $2 \%$ no matter a changing $\mathrm{r}$ or $L$, and although the explosion distance is rather short, the error value is not more than $2 \%$, so the approximate formula for the first shadow condition is accurate.

To verify the accuracy of the approximate theoretical formula for the second shadow condition, only the explosion position and the longitudinal length of the cylinder are changed. The cylinder diameter is set as $5.5 \mathrm{~m}$, and the corresponding angle is set as $\theta^{\prime}=0.3105$. The explosive along the length of the cylinder remains unchanged; in any cross section of the hull, transforming the explosive along the breadth and draft direction changes the explosion distance, the specific conditions are shown in Fig. 3.13: 


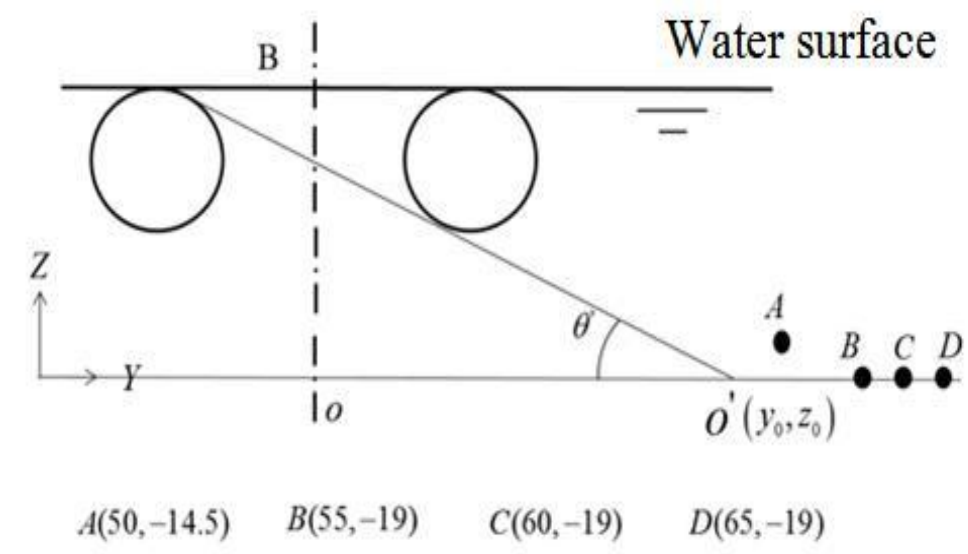

Fig. 3.13 Schematic diagram of the explosive on any cross section of the ship

Table $3.4 \mathrm{~T}=5.5 \mathrm{~m}$ the error value corresponding to different $\mathrm{r} / \mathrm{L}, \mathrm{R}$ (unit:\%)

\begin{tabular}{|c|c|c|c|c|c|c|c|c|c|c|}
\hline \multirow[t]{2}{*}{ Vertical } & \multicolumn{10}{|c|}{$r / L$} \\
\hline & 5.45 & 7.27 & 9.09 & 10.91 & 12.73 & 14.55 & 16.36 & 18.18 & 20.00 & 21.82 \\
\hline 40.0 & & & & & & & & & & \\
\hline$\theta / \theta^{\prime}=0.77$ & 8.0 & 8.1 & 8.3 & 8.4 & 8.6 & 8.7 & 8.9 & 8.6 & 8.8 & 8.9 \\
\hline 46.4 & & & & & & & & & & \\
\hline$\theta / \theta^{\prime}=0.98$ & 0.2 & 0.1 & 0.1 & 0.2 & 0.1 & 0.0 & 0.0 & 0.2 & 0.1 & 0.0 \\
\hline 51.1 & & & & & & & & & & \\
\hline$\theta / \theta^{\prime}=0.90$ & 3.1 & 3.2 & 3.2 & 3.2 & 3.3 & 3.4 & 3.5 & 3.1 & 3.2 & 3.3 \\
\hline 55.8 & & & & & & & & & & \\
\hline$\theta / \theta=0.83$ & 6.0 & 6.1 & 6.1 & 6.1 & 6.3 & 6.3 & 6.4 & 5.9 & 6.0 & 6.1 \\
\hline
\end{tabular}




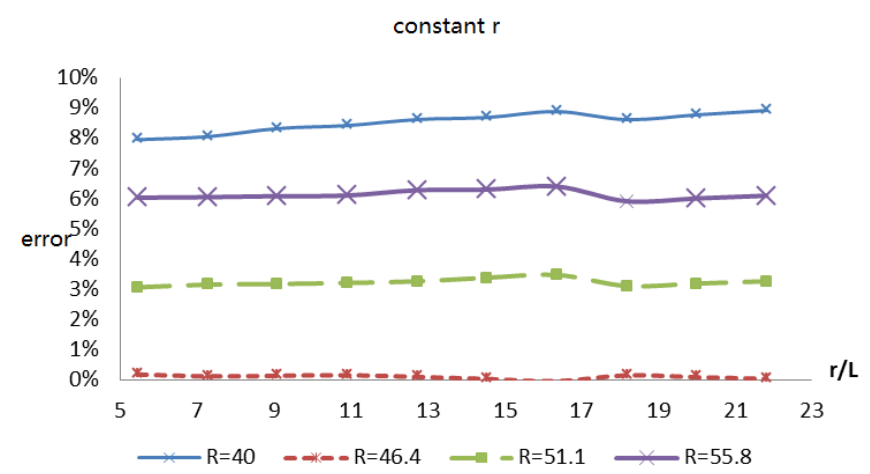

Fig. 3.14 Diagram of the error changing with $\mathrm{r} / \mathrm{L}$

As shown in Fig. 3.14, the error remains almost the same; the r/L changes when the explosion distance remains the same, but it appeared as an irregular change when the explosion distance is moved but the $\mathrm{r} / \mathrm{L}$ is not changed. Obviously, this indicates that the error increases with the decrease of $\theta / \theta$; the maximum is $9 \%$, so the error changes with $\theta / \theta^{\prime}$. In order to improve the accuracy of the formula, verification of the $S_{e 2}$ is needed. The specific result is shown in Table 3.5.

Table 3.5 Contrast between theoretical approximate result and standard value

\begin{tabular}{|c|c|c|c|c|c|c|c|c|c|}
\hline$\theta / \theta^{\prime}$ & 0.98 & 0.94 & 0.90 & 0.85 & 0.81 & 0.76 & 0.72 & 0.67 & 0.63 \\
\hline $\mathrm{R}$ & 53.33 & 52.96 & 52.60 & 52.26 & 51.94 & 51.63 & 51.34 & 51.07 & 50.81 \\
\hline$S_{e 1}$ & 373.8 & 365.7 & 357.7 & 349.6 & 341.5 & 333.4 & 325.4 & 317.3 & 309.3 \\
\hline$S_{e 2}$ & 372.7 & 371.6 & 370.4 & 369.3 & 368.1 & 367.5 & 366.1 & 365.3 & 364.4 \\
\hline error & & & & & & & & & \\
\hline$(\%)$ & 0.003 & 0.02 & 0.03 & 0.05 & 0.07 & 0.09 & 0.11 & 0.13 & 0.15 \\
\hline
\end{tabular}




\begin{tabular}{|c|c|c|c|c|c|c|c|c|c|}
\hline$\theta / \theta^{\prime}$ & 0.58 & 0.53 & 0.49 & 0.44 & 0.39 & 0.35 & 0.30 & 0.25 & 0.20 \\
\hline $\mathrm{R}$ & 50.57 & 50.35 & 50.15 & 49.96 & 49.80 & 49.65 & 49.52 & 49.41 & 49.32 \\
\hline$S_{e 1}$ & 301.3 & 293.3 & 285.3 & 277.4 & 269.5 & 261.5 & 253.7 & 245.8 & 238.0 \\
\hline$S_{e 2}$ & 363.5 & 362.5 & 361.8 & 361.1 & 360.4 & 359.8 & 359.6 & 359.3 & 358.9 \\
\hline error & & & & & & & & & \\
\hline$(\%)$ & 0.17 & 0.19 & 0.21 & 0.23 & 0.25 & 0.27 & 0.29 & 0.32 & 0.34 \\
\hline
\end{tabular}

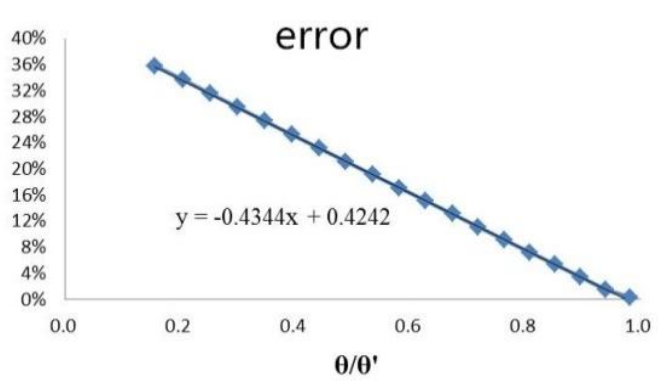

(a) The curve of the error

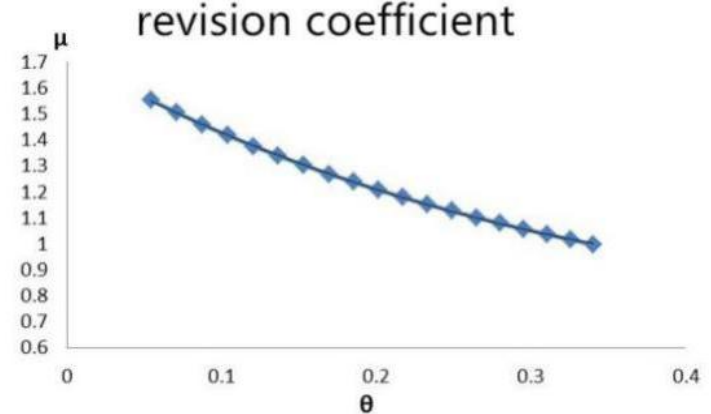

(b) The curve of revision

Fig. 3.15 Diagram of the error changing with $\theta / \theta^{\prime}$

As Fig. 3.15 (a) shows, when $\theta / \theta^{\prime}$ gradually decreases, this indicates an increase in the shadow area of the left cylinder that is sheltered by the right cylinder. So the error of the approximate theoretical result is significant and reaches a maximum of $34 \%$. Such a great error is not allowed in the project application. Hence, the formula 
needs the revision coefficient $\mu$. From the function generated by the curve in Fig. $3.15(b)$, there is:

$$
\frac{S_{e 2}-S_{e 1}}{S_{e 2}}=k \frac{\theta}{\theta^{\prime}}+b
$$

Then:

$$
\begin{gathered}
S e_{2}=\frac{\theta^{\prime}}{(1-b) \theta^{\prime}-k \theta} \cdot S e_{1}=\mu \cdot S e_{1} \\
\mu=\frac{\theta^{\prime}}{\left(1-b \theta^{\prime}-k \theta\right.}
\end{gathered}
$$

If the model is determined, then the parameters $k, b, \theta, \theta^{\prime}$ are also determined. According to equation (14), the revision coefficient $\mu-\theta$ curve shown in Fig. 3.15 (b) can be obtained.

The shock factor of the first condition is defined as follows:

$$
C_{\text {catal }}=\left\{W\left[\begin{array}{c}
\arcsin \left(\frac{r}{r+R}\right)\left(\sin \left(\arctan \frac{\Delta l_{2}}{R}\right)+\sin \left(\arctan \frac{\Delta l_{1}}{R}\right)\right)+ \\
\arcsin \left(\frac{r}{r+R^{\prime}}\right)\left(\sin \left(\arctan \frac{\Delta l_{2}}{R^{\prime}}\right)+\sin \left(\arctan \frac{\Delta l_{1}}{R^{\prime}}\right)\right)
\end{array}\right]\right\}^{0.5}
$$

The shock factor of the second condition is defined as follows:

$$
C_{\text {cata } 2}=\left\{W \mu\left[\begin{array}{l}
\arcsin \left(\frac{r}{r+R}\right)\left(\sin \left(\arctan \frac{\Delta l_{2}}{R}\right)+\sin \left(\arctan \frac{\Delta l_{1}}{R}\right)\right)+ \\
\left.\left(\arctan \left|\frac{z_{e}-z_{0}}{y_{e}-y_{0}}\right|-\arctan \left|\frac{z_{f}-z_{0}}{y_{f}-y_{0}}\right|\right)\left(\sin \left(\arctan \frac{\Delta l_{2}}{R^{\prime}}\right)+\sin \left(\arctan \frac{\Delta l_{1}}{R^{\prime}}\right)\right)\right]
\end{array}\right\}^{0.5}\right.
$$




\section{The description and result of the numerical experiment}

The total kinetic energy and velocity response are two physical parameters that are closely related to the impact environment of a ship's hull. The total strain energy is the response caused by the deformation of ship hull vibration [15]. Therefore, the total kinetic energy, strain energy and speed response are the most direct manifestations of the ship impact environment. In order to examine the validity of the new shock factor, an equal shock factor condition is designed in this section, and this section also separately analyzes the similarity of the impact environment with regard to three aspects, including the total kinetic energy, the total potential energy and vertical shock spectrum.

\subsection{The verification of the first shadow condition}

The equal shock factor condition was designed with regard to the first shadow condition. The midship section is shown in Fig. 4.1. The explosive is $20 \mathrm{~m}$ to $100 \mathrm{~m}$ below the surface of the water; the explosive quality is increased from $10.6 \mathrm{Kg}$ to 167.1 Kg. The specific parameters are shown in Table 4.1.

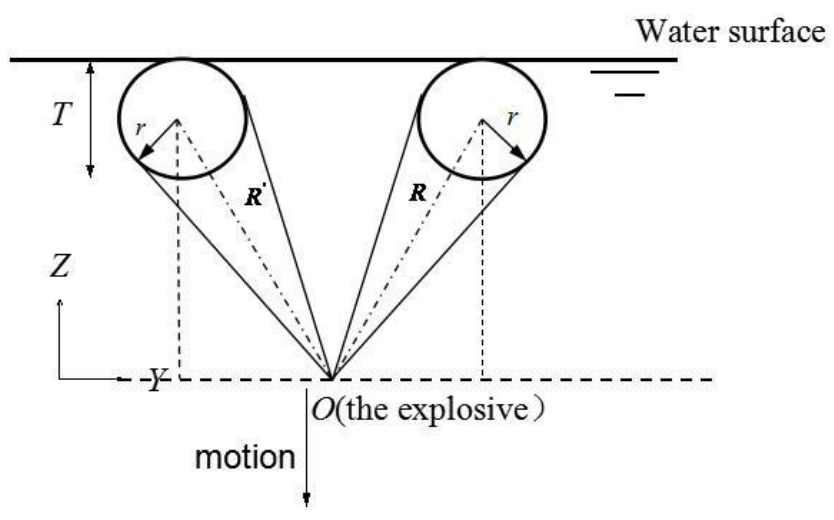

Fig. 4.1 The position of the explosive 
Table 4.1 The verification of the first shadow condition

\begin{tabular}{c|c|c|c|c|c|c|c|c|c}
\hline Condition number & $1-1$ & $1-2$ & $1-3$ & $1-4$ & $1-5$ & $1-6$ & $1-7$ & $1-8$ & $1-9$ \\
\hline $\begin{array}{c}\text { The weight of } \\
\text { explosive W (Kg) }\end{array}$ & 10.6 & 18.7 & 39.6 & 44.0 & 61.8 & 83 & 107.6 & 135.6 & 167.1 \\
\hline $\begin{array}{c}\text { Explosive distance } \\
\text { R (m) }\end{array}$ & 14.6 & 24.6 & 34.5 & 44.5 & 54.5 & 64.5 & 84.5 & 84.5 & 94.5 \\
\hline Distance to water & 20 & 30 & 40 & 50 & 60 & 70 & 80 & 90 & 100 \\
surface & & & & & & & & & \\
\hline
\end{tabular}

The hull's total kinetic energy and the total potential energy curves using the verification condition are shown in Fig. 4.2 (a) and (b). The curves of the above conditions are essentially coincident, which indicates that the ship response is almost the same as under equal shock factor conditions.

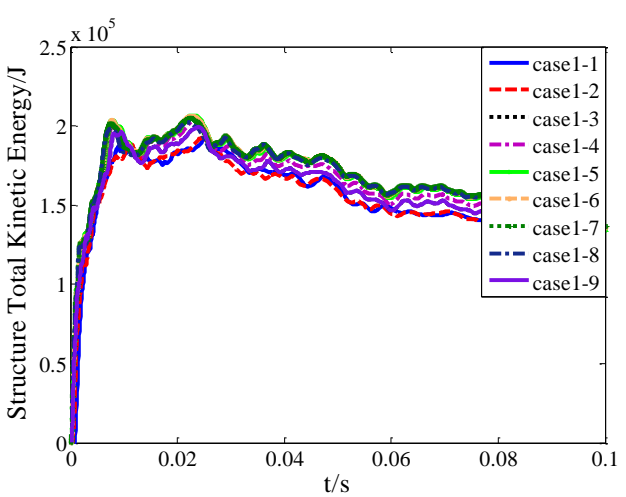

(a) Time history curves of the total

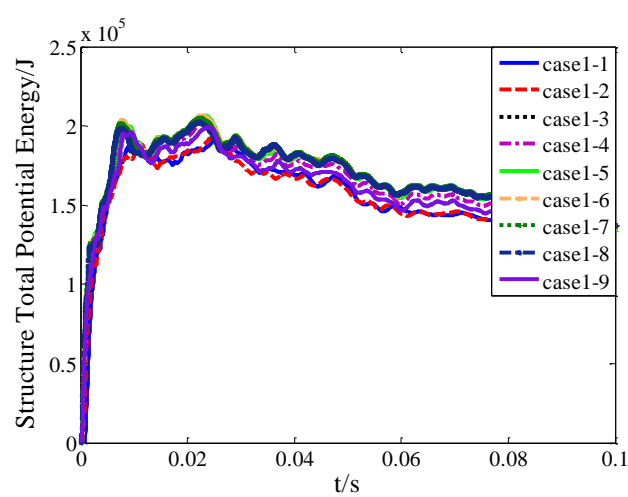

(b) Time history curves of the total 
Fig. 4.2 Curves of ship energy in a verification condition

Certain points in the superstructure, bulwark and on the bottom of the hull were picked and checked to analyze specifically whether the responses of the structure are consistent. The shock spectrum results are shown in Fig. 4.2.

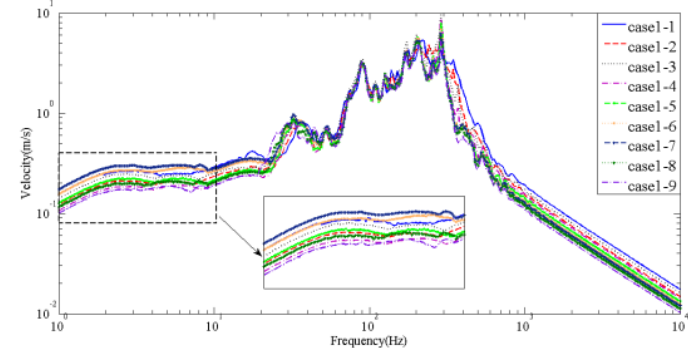

(a) Checkpoints on the bottom

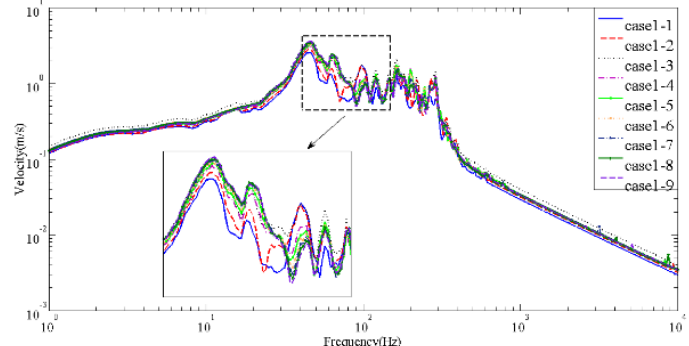

(b) Checkpoints in bulwark

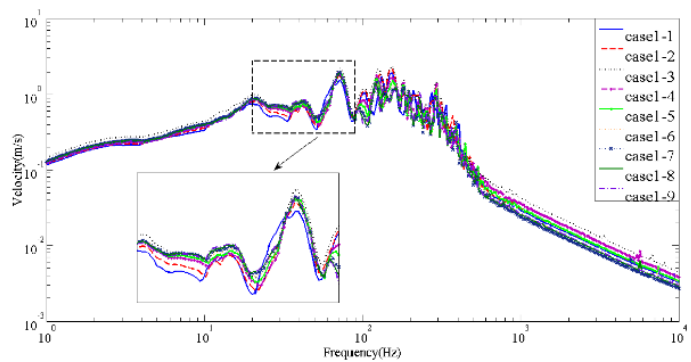

(c) Checkpoints in superstructure

Fig. 4.3 Diagrams of shock spectrums in verification conditions

As shown in Fig. 4.3, the shock responses of the checkpoints are nearly consistent, and the checkpoints in bulwark are consistently the best. This indicates that the shock factor in the first shadow condition is precise.

4.2 The verification of the second shadow condition 
The equal shock factor condition was designed and the explosive location was changed with regard to the second shadow condition. The specific parameters are shown in Table 4.2 and Table 4.3:

Table 4.2 the specific parameters of the condition

\begin{tabular}{c|c|c|c|c|c|c|c|c}
\hline Condition number & $2-1$ & $2-2$ & $2-3$ & $2-4$ & $2-5$ & $2-6$ & $2-7$ & $2-8$ \\
\hline Y value of the & 25 & 30 & 40 & 50 & 50 & 55 & 60 & 65 \\
explosive & -11 & -15 & -15 & -15 & -20 & -20 & -20 & -20 \\
\hline Z value of the & & & & & & & & \\
explosive & 16.1 & 21.4 & 31.4 & 40.9 & 42.6 & 47.2 & 52 & 56.7 \\
\hline Explosive distance & & & & & & & & \\
\hline R (m) & & & & & & & & \\
\hline
\end{tabular}

Table 4.3 the verification of the second shadow condition

\begin{tabular}{c|c|c|c|c|c|c|c|c}
\hline Condition number & $2-1$ & $2-2$ & $2-3$ & $2-4$ & $2-5$ & $2-6$ & $2-7$ & $2-8$ \\
\hline$\theta$ (radian) & 0.32 & 0.33 & 0.30 & 0.24 & 0.33 & 0.30 & 0.28 & 0.26 \\
\hline Revision coefficient $\mu$ & 1.03 & 1.01 & 1.05 & 1.14 & 1.01 & 1.05 & 1.08 & 1.11 \\
\hline The weight of & 8.4 & 11.5 & 19.9 & 29.45 & 31.3 & 36.9 & 43.2 & 50 \\
\hline
\end{tabular}




\begin{tabular}{c|c|c|c|c|c|c|c|c}
\hline explosive W (Kg) & & & & & & & & \\
\hline explosive distance R (m) & 16.1 & 21.4 & 31.4 & 40.9 & 42.6 & 47.2 & 52.0 & 56.7 \\
\hline
\end{tabular}

The curves of the total kinetic energy and total potential energy are shown in Fig.

4.4 .

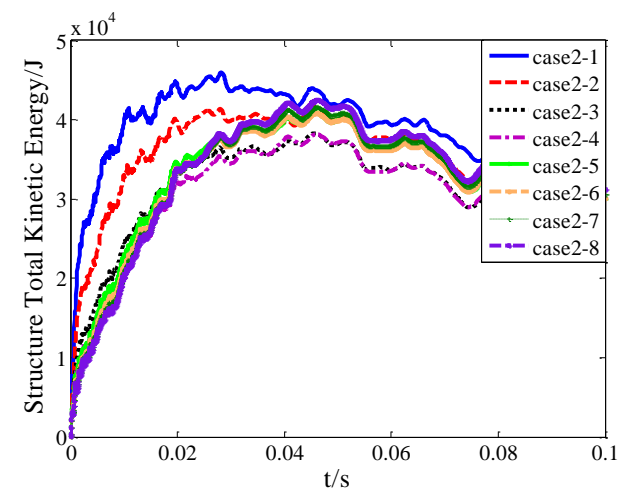

(a)Time history curve of the total kinetic energy

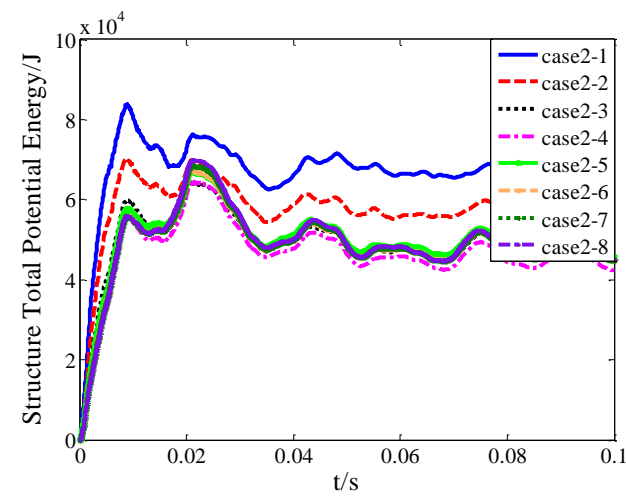

(b) Time history curve of the total potential energy

Fig. 4.4 Curves of ship energy in verification condition

Fig. 4.4 only marks the curves of the $2-1$ and $2-2$ conditions, the others are 2-3 2-8 conditions, and these curves are almost consistent, as shown in Fig. 4.4 (a). The curves of the 2-1 and 2-2 conditions are obviously higher than others; the kinetic energy of $2-1$ condition is 1.13 times more than that of the $2-8$ condition, and the potential energy is 1.5 times that. This phenomenon shows that the precision of the shock factor is relatively poor if the explosion distance is less than $20 \mathrm{~m}$, while it will be more accurate if the explosion distance is more than $20 \mathrm{~m}$. 


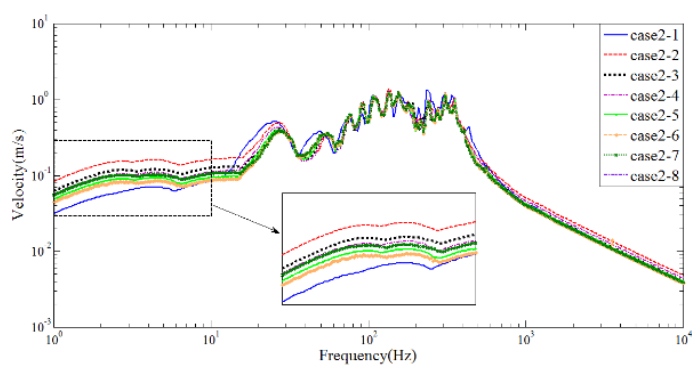

(a) Checkpoints on the bottom

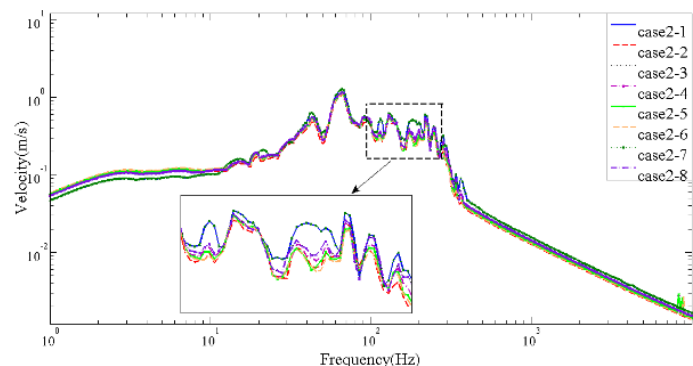

(b) Checkpoints in bulwark

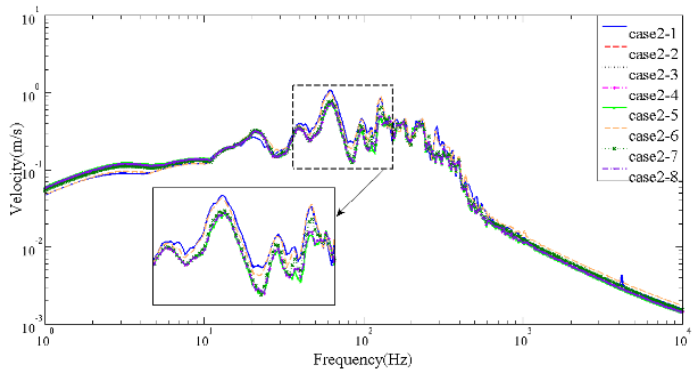

(c) Checkpoints in superstructure

Fig. 4.5 The diagram of shock spectrum in verification conditions

The diagrams of shock spectrum in verification conditions are shown in Fig. 4.5, showing that the curves of the shock spectrum for different checkpoints are almost consistent. Only at the bottom is there little difference in the low-frequency region. Therefore, the shock factor of the second condition is also very precise.

\section{Conclusions}

1) When calculating the underwater shadow area of the SWATH catamaran, the simplified cylinder model with a diameter that is equal to its cylinder can be used instead of the complicated underwater structure one.

2) The shock factor of the SWATH catamaran can be divided into two types based on the shadow conditions. 
3) The shock factor of the first shadow condition demonstrates good precision in the description of impact environment of the ship subjected to an underwater explosion. The equal curves of the same shock factor conditions that are shown on the shock factor spectrum are consistent. This indicates that it can describe the similarity of the impact environment also when in this condition.

4) The accuracy of the approximate theoretical calculation formula for the shadow area decreases as the value of $\theta / \theta^{\prime}$ decreases in the second shadow condition. This means it needs to be revised using the finite element method; the shock factor is then more precise, but its precision is not as good as that of the first shadow condition.

\section{Acknowledgments}

This study is supported by grants $(51009035,51479041,51279038)$ from the

National Science Foundation of China and a China Postdoctoral Science Foundation Grant (2014T70311).

\section{References}

[1] Shi Wen-qiang, Yu Xian-zhao. The development status and trend of SWATH catamaran in foreign [J]. Ship science and technology, 2012,34(02): 4-5.

[2] Van Sluijs, M. F. Model and full scale motions of a twin-hull vessel[A]. Netherlands Ship Research Center TNO, 1969, 21 pp.

[3] Lang, T. G., J. D, Hightower, A. T. Strickland. Design and development of the 190-ton stable semisubmerged plat-form (SSP)[A]. Engineering for Industry, 
Nov. 1974, pp.1105-1111.

[4] Hightower, J. D., R. L. Steiple. Operational experiences with the SWATH ship SSP KAIMALINO[A], AIAA/ SNAME Advanced Marine Vehicle Conf, Apr. 1978, Paper 78-741.

[5] COLE R H. Underwater Explosion [M].New Jersey: Princeton University Press, 1948.

[6] ZHANG Zhen-hua. Study on explode-resistant of naval vessels subjected to underwater explosion [D]. Wuhan: Naval University of Engineering, 2004.

[7] Yao Xiong-liang, Cao Yu, Guo Jun, Xie Jian-lin. An explosive shock factor under water [D]. Journal of Harbin Engineering University, 2007, 28(05).

[8] Guo Jun. Research on characteristics of impact environment of stiffened double cylindrical shell [D]. Master degree thesis of Harbin Engineering University, 2005,63-64.

[9] Cao Yu. The similarity research of explosive shock environment under water [D]. Master degree thesis of Harbin Engineering University, 2006, 23-29.

[10] Bai Zhao-hong. Modeling method for underwater explosion shock environment in the far field [D]. Master degree thesis of Harbin Engineering University, 2012, $16-32$

[11] Gu Wang-ming, Huang Jun-de. Analysis of cylindrical shell pressure subjected to underwater explosion shock wave wall [J]. Journal of Naval Engineering College, 
1989, (49): 14-22.

[12] A.M. Zhang, Y.L. Liu. Improved three dimensional bubble dynamics model based on boundary element method. Journal of Computational Physics, 2015, 294: 208-223.

[13] Zhang A-man, Yang Wen-shan, Yao Xiong-liang. Numerical simulation of underwater contact explosion [J]. Applied Ocean Research, 2012, 34:10-20.

[14] HUN G C F, HSU P Y, HWA GFUU J J. Elastic shock response of an air-backed plate to underwater explosion [J]. International Journal of Impact Engineering, 2005, 31: $151-168$.

[15] A. M. Zhang, P. Cui, J. Cui, Q. X. Wang. Experimental study on bubble dynamics subject to buoyancy [J]. Journal of Fluid mechanics, 2015. 